\title{
Silencing of ASPP2 promotes the proliferation, migration and invasion of triple-negative breast cancer cells via the PI3K/AKT pathway
}

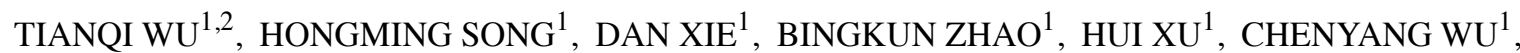 \\ KAIYAO HUA ${ }^{1}$, YIJUN DENG ${ }^{1,2}$, CHANGLE $\mathrm{JI}^{1}$, JIASHU HU ${ }^{1}$ and LIN FANG ${ }^{1}$
}

${ }^{1}$ Department of Breast and Thyroid Surgery, Shanghai Tenth People's Hospital, Tongji University School of Medicine, Shanghai 200072; ${ }^{2}$ The First School of Clinical Medicine, Nanjing Medical University, Nanjing, Jiangsu 210029, P.R. China

Received September 15, 2017; Accepted March 8, 2018

DOI: $10.3892 /$ ijo.2018.4331

\begin{abstract}
Apoptosis-stimulating p53 protein 2 (ASPP2) is an apoptosis inducer that acts via binding with p53 and then enhancing the transcriptional activities toward pro-apoptosis genes. ASPP2 has recently been reported to serve a major role in p53-independent pathways. Triple-negative breast cancer (TNBC) is a type of breast cancer that is more aggressive and highly lethal when p53 is mutated. In the present study, the mRNA level of ASPP2 was found to be suppressed in breast tumors compared with that in adjacent normal breast tissues, and the expression of ASPP2 was also decreased in a series of breast cancer cell lines compared with that in MCF-10A normal breast cells. Downregulation of ASPP2 by specific small interfering RNA (siRNA) transfection was able to promote cell growth, reduce cell apoptosis, and contribute to cell migration and invasion. Furthermore, downregulation of ASPP2 promoted cell epithelial-mesenchymal transition (EMT) in MDA-MB-231 and HCC-1937 TNBC cells. Furthermore, it was found that when ASPP2 siRNA was transfected into MDA-MB-231 and HCC-1937 cells, the expression of phosphoinositide-3-kinase regulatory subunit $1(\mathrm{p} 85 \alpha)$ decreased and phosphorylation of protein kinase B (AKT) increased, which are key molecular regulators in the phosphatidylinositol 3-kinase (PI3K)/AKT pathway. In conclusion, the present data indicated that ASPP2 had a crucial influence on the proliferation and metastasis in TNBC, and that the functional mechanism may be p53-independent to a great extent. ASPP2 and its link with the PI3K/AKT pathway deserve further investigation and may provide novel insights into therapeutic targets for TNBC.
\end{abstract}

Correspondence to: Professor Lin Fang or Dr Tianqi Wu, Department of Breast and Thyroid Surgery, Shanghai Tenth People's Hospital, Tongji University School of Medicine, 301 Yanchang Road, Jing'an, Shanghai, P.R. China

E-mail: fanglin2017@126.com

E-mail:wtqttkk@126.com

Key words: ASPP2, phosphatidylinositol 3-kinase/protein kinase B, triple-negative breast cancer, epithelial-mesenchymal transition

\section{Introduction}

Breast cancer is one of the most prevalent cancer types among females worldwide. In addition to the sharp increase in the rate of morbidity, the average age of morbidity has gradually decreased (1). Triple-negative breast cancer (TNBC) is a clinical phenotype characterized by the lack of three proteins: Estrogen receptor, progesterone receptor and human epidermal growth factor receptor 2 (HER2). TNBC tends to be more aggressive and has a higher mortality rate compared with the other breast cancer subtypes (2). As a result of the deficiency of certain receptors, hormone therapy and anti-HER 2 targeted therapy are ineffective. Treatment primarily relies on chemotherapy (3). However, treatment failure has become a growing trend, accompanied by tumor relapse and chemotherapeutic resistance (4). Consequently, there is an urgent requirement to ascertain the precise molecular mechanisms of TNBC and seek novel strategies for its treatment.

Apoptosis-stimulating p53-binding protein 2 (ASPP2), also known as 53BP2L, is the long form of the two splicing variants encoded by the tumor protein p53 binding protein 2 gene through alternative splicing (5). ASPP2 was originally identified as an activator of the p53 family of proteins that specifically enhanced their transcriptional activities toward pro-apoptosis genes (but not genes in association with cell-cycle arrest) by binding to them $(6,7)$. However, emerging evidence has suggested that ASPP2 is associated with a series of p53-independent biological pathways, rather than simply inducing apoptosis dependent on p53 (8). One study indicated that ASPP2 could promote Ras-induced senescence through the direct interaction of its N-terminus with Ras-GTP (9). Furthermore, it serves as a pivotal regulator of cell polarity and the autophagy process $(10,11)$. ASPP2 has also been confirmed to bind and co-localize with PAR3, thereby inhibiting tumor metastasis as a molecular switch of epithelial-mesenchymal transition (EMT), and the reduction of ASPP2 results in the poor survival and prognosis of patients with hepatocellular carcinoma and breast cancer (12). In the majority of human cancer types, including hepatocellular carcinoma (13), pancreatic cancer and cervical cancer $(14,15)$, ASPP2 is considered to be a tumor suppressor, usually with low expression. Notably, 
widespread p53 mutations take place in TNBC (16), which may limit the role of ASPP2 with regard to p53-dependent pro-apoptosis, since it acts only on wild type-p53 genes rather than mutant-p53 genes $(17,18)$. The mechanisms and functions of ASPP2 in the presence of p53 mutations are much less well known, particularly in TNBC, in which p53 mutation frequently occurs.

In the present study, the function and associated mechanisms of ASPP2 in TNBC were investigated. The aims of the present study were to examine the relative expression of ASPP2 in breast cancer samples and cell lines, to investigate its functional roles in cell proliferation, migration and invasion using specific small interfering RNA (siRNA), and to investigate the possibility of its target signaling pathways as potential molecular targets for therapeutic agents.

\section{Materials and methods}

Patients and samples. The breast tumor tissues and paired normal adjacent tissues were collected from patients who underwent surgical resection at the Department of Breast and Thyroid Surgery of the Shanghai Tenth People's Hospital (Shanghai, China) between December 2016 and February 2017. The patients were women between the ages of 32 and 71 years, with a mean age of 53 years. None of the patients had received any chemotherapy (19) or radiotherapy prior to surgery. Patients with distant metastases or a history of a previous or concomitant malignancy were excluded. The samples were immediately snap-frozen in liquid nitrogen. Tumor and normal tissues were histologically confirmed by more than one experienced pathologist according to the World Health Organization guidelines (19), using hematoxylin and eosin staining. All specimens were embedded in $10 \%$ formalin solution for $12-24 \mathrm{~h}$ at room temperature and cut into $5-\mu \mathrm{m}$ thick sections. The sections were stained with hematoxylin for 3-10 min and with eosin for $60 \mathrm{sec}$ at room temperature, and then observed under a light microscope at $\mathrm{x} 40$ magnification. The specimen collection and use was approved by the Institutional Ethics Committees of Tongji University (Shanghai, China). All patients provided written informed consent. The data of the patients are not shown.

Cell culture and reagent. Human breast cancer cell lines, MDA-MB-231, HCC-1937, MCF-7, BT-549 and MDA-MB-468, and the human mammary epithelial cell line, MCF-10A, were purchased from the Chinese Academy of Sciences (Shanghai, China). The BT-549 cells were cultured in RPMI-1640 medium (Gibco; Thermo Fisher Scientific, Inc., Waltham, MA, USA) supplemented with $100 \mathrm{U} / \mathrm{ml}$ penicillin, $100 \mu \mathrm{g} / \mathrm{ml}$ streptomycin (Enpromise, Hangzhou, China) and 10\% fetal bovine serum (FBS; Gibco; Thermo Fisher Scientific, Inc.). MCF-10A cells were cultured in mammary epithelial basal medium (Cambrex Corporation, East Rutherford, NJ, USA). The remaining cells were grown in Dulbecco's modified Eagle's medium (DMEM) supplemented with 10\% FBS (both Gibco; Thermo Fisher Scientific, Inc.), penicillin (100 U/ml) and streptomycin $(100 \mu \mathrm{g} / \mathrm{ml})$ (both from Enpromise). All cells were incubated at $37^{\circ} \mathrm{C}$ in a humidified chamber containing $5 \% \mathrm{CO}_{2}$.

ASPP2 siRNA and negative control siRNA (NC siRNA) oligonucleotides were chemosynthesized by Sangon Biotech Co.,
Ltd. (Shanghai, China). The sequence of the ASPP2 siRNA was 5'-GCCCAGUAGAAAUCCAGAATT-3' (sense) and 5'-UUC UGGAUUUCUACUGGGCTT-3' (antisense), while the sequence of the NC siRNA was 5'-UUCUCCGAACGUGUCACGUTT-3' (sense) and 5'-ACGUGACACGUUCGGAGAATT-3' (antisense).

Transfection assay. The MDA-MB-231, MCF-7 and HCC-1937 cells $\left(8 \times 10^{4} /\right.$ well $)$ were cultured in a 6 -well plate with serum and antibiotic-free DMEM for transfection. When the confluence reached 30-50\%, transfection of ASPP2 siRNA and NC siRNA was performed using the Lipofectamine ${ }^{\circledR} 2000$ Transfection kit (Invitrogen; Thermo Fisher Scientific, Inc.), according to the manufacturer's protocols, at working concentrations. The concentration of siRNAs used was $100 \mathrm{nmol} / \mathrm{l}$, and the ratio of mimics to Lipofectamine 2000 was 1.25:1.00 (volume). The medium was replaced by DMEM with 10\% FBS after 4-6 h of incubation. The cells were used for future analysis after $48 \mathrm{~h}$ of transfection.

Reverse transcription-quantitative polymerase chain reaction $(R T-q P C R)$ assay. Total cellular RNA was extracted from the transfected MDA-MB-231, MCF-7 or HCC-1937 cells using TRIzol (Invitrogen; Thermo Fisher Scientific, Inc.) and stored at $-80^{\circ} \mathrm{C}$. For ASPP 2 detection, cDNA was generated by RT using the PrimeScript RT-PCR kit (Takara Bio, Inc., Otsu, Japan) in accordance with the manufacturer's protocols. Conditions of the RT reaction were $37^{\circ} \mathrm{C}$ for $15 \mathrm{~min}$, then $85^{\circ} \mathrm{C}$ for $5 \mathrm{sec}$. RT-qPCR was performed using SYBR-Green PCR master mix (Takara Bio, Inc.) on a 7900HT Fast RT-PCR instrument (Applied Biosystems; Thermo Fisher Scientific, Inc.). The amplification procedure was as follows: $3 \mathrm{~min}$ at $95^{\circ} \mathrm{C}$, followed by 40 cycles at $95^{\circ} \mathrm{C}$ for $3 \mathrm{sec}$ and $60^{\circ} \mathrm{C}$ for $30 \mathrm{sec}$. The relative expression was evaluated following the relative quantification $2^{-\Delta \Delta C t}$ method (20). Each sample was tested in triplicate. The primers used in the RT-PCR were as follows: ASPP2 forward, 5'-CTGTGCAAA GAACCCGGCG-3' and reverse, 5'-CAACTGGACGTTCAG AGCCACA-3'; and $\beta$-actin forward, 5'-CAGAGCCTCGCC TTTGCC-3' and reverse, 5'-GTCGCCCACATAGGAATC-3'.

Western blot assay. The transfected MDA-MB-231, MCF-7 or HCC-1937 cells were harvested and lysed in radioimmunoprecipitation assay lysis buffer ( $80 \mu 1 /$ well; Beyotime Institute of Biotechnology, Jiangsu, China) after 48-72 h of transfection. The protein concentration was quantified with a bicinchoninic acid protein assay kit (Beyotime Institute of Biotechnology). Next, equal amounts of protein $(30-50 \mu \mathrm{g})$ were separated by 8 or $10 \%$ sodium dodecyl sulfate-polyacrylamide gel electrophoresis (Beyotime Institute of Biotechnology), and then transferred to $0.45-\mu \mathrm{m}$ nitrocellulose membranes using the cold transfer buffer $(3.03 \mathrm{~g}$ Tris $+14.4 \mathrm{~g}$ glycine $+200 \mathrm{ml}$ methanol $+800 \mathrm{ml}$ deionized water). Subsequent to blocking at room temperature for $1 \mathrm{~h}$ in $5 \%$ skimmed milk diluted with phosphate-buffered saline plus Tween-20 (PBST), the membranes were hybridized overnight at $4^{\circ} \mathrm{C}$ with specified primary antibodies in PBST containing 5\% skimmed milk. Subsequently, the membranes were washed with PBST and incubated with IRDye 680 donkey anti-mouse IgG-(H+L) (1:1,000 dilution; cat. no. 926-68072) or goat anti-rabbit IRDye $800 \mathrm{CW}$ secondary antibody (1:1,000 dilution; cat. no. 926-32211; LI-COR Biosciences, Lincoln, NE, USA) 
for $1 \mathrm{~h}$ at a room temperature. Protein bands were detected with an Odyssey Scanning system (LI-COR Biosciences).

Antibodies used were follows: Anti-ASPP2 (1:20,000 dilution; cat. no. ab181377; Abcam, Cambridge, UK), anti- $\beta$-actin (1:2,000 dilution; cat. no. sc-47778; Santa Cruz Biotechnology, Inc., Dallas, TX, USA), anti-caspase-9 (1:1,000 dilution; cat. no. ab202068; Abcam), anti-caspase-3 (1:1,000 dilution; cat. no. 9662; Cell Signaling Technology, Inc., Danvers, MA, USA), anti-poly (ADP-ribose) polymerase (PARP; 1:1,000 dilution; cat. no. ab191217; Abcam), anti-Bax (1:1,000 dilution; cat. no. 2772), anti-E-cadherin (1:750 dilution; cat. no. 3195) (both from Cell Signaling Technology, Inc.), anti-N-cadherin (1:2,000 dilution; cat. no. ab18203), anti-Snail (1:1,000 dilution; cat. no. ab82846), anti-zinc finger E-box-binding homeobox 1 (ZEB1; 1:1,000 dilution; cat. no. ab155249), anti-matrix metalloproteinase 2 (MMP2; 1:2,000 dilution; cat. no. ab37150) (all from Abcam), anti-MMP9 (1:1,000 dilution; cat. no. 54980; Arigo Biolaboratories, Hsinchu, Taiwan), anti-phosphoinositide-3-kinase regulatory subunit 1 (PIK3R1; 1:1,000 dilution; cat. no. 13666), anti-AKT (1:1,000 dilution; cat. no. 9272), anti-phosphorylated (p-)AKT (ser-473; 1:1,000 dilution; cat. no. 4060) (all from Cell Signaling Technology, Inc.), anti-extracellular signal-regulated kinases (ERK; 1:2,000 dilution; cat. no. ab17942) and anti-p-ERK (ser-T202 and ser-T185; 1;1,000 dilution; cat. no. ab201015) (both from Abcam).

MTT assay. At $24 \mathrm{~h}$ post-transfection, the MDA-MB-231 and HCC-1937 cells were seeded in $200 \mu \mathrm{l}$ growth medium at $5 \times 10^{2}$ cells per well in 96-well plates (BD Biosciences, Franklin Lakes, NJ, USA) and incubated overnight at $37^{\circ} \mathrm{C}$ in $5 \% \mathrm{CO}_{2}$. Every 24 h until 72 h, $20 \mu \mathrm{l}$ MTT (Sigma-Aldrich; Merck KGaA, Darmstadt, Germany) solution was added to each well and incubated at $37^{\circ} \mathrm{C}$ for $4 \mathrm{~h}$. Next, $150 \mu \mathrm{l}$ dimethyl sulfoxide (Sigma-Aldrich; Merck KGaA) was added to each well and agitated gently for $10 \mathrm{~min}$ to dissolve the MTT formazan crystals after removing the supernatant. Cell viability was measured by the recording absorbance at $490 \mathrm{~nm}$ with a microplate reader (BioTek Instruments, Inc., Winooski, VT, USA).

Colony formation assay. A total of $1 \times 10^{3}$ transfected MDA-MB-231 and HCC-1937 cells from each group were seeded in a 6-well plate in DMEM with $10 \%$ FBS. The plates were agitated to disperse the cells equally. After 7 to 10 days of culturing, or when the colonies were visible, the cell culture was terminated and the plates were washed twice with PBS. Next, the cells were fixed in $95 \%$ ethanol for $10 \mathrm{~min}$, dried and stained with $0.1 \%$ crystal violet solution for $10 \mathrm{~min}$ at room temperature. Finally, the staining solution was washed away and the number of colonies with diameters of $>1.5 \mathrm{~mm}$ was counted by eye. The experiments were performed in triplicate.

Wound-healing assay. To assay the migratory response of breast cancer cells to ASPP2 expression, the transfected MDA-MB-231 and HCC-1937 cells were seeded into 6-well plates and cultured until the cells reached $\sim 90 \%$ confluence. Next, a scratch was made in each well using a sterile pipette tip. Cells were washed with PBS to remove cellular debris and allowed to migrate for $48 \mathrm{~h}$. The process of wound healing was observed under a light microscope and representative images were acquired at 0 and $48 \mathrm{~h}$ post-wounding with a digital camera system. All experiments were performed in triplicate.

Transwell invasion assay. The transfected MDA-MB-231 and HCC-1937 cells at a density of $5 \times 10^{4}$ were suspended in serum-free DMEM $(200 \mu \mathrm{l})$ and added into the upper chamber of the Transwell, with a Matrigel-coated $(2 \mathrm{mg} / \mathrm{ml})$ membrane containing $8-\mu \mathrm{m}$ diameter pores, to observe invasion following transfection. Complete DMEM (500 $\mu \mathrm{l})$ was then added to the bottom chamber of 24 -well plates to serve as a chemoattractant. Subsequent to $20 \mathrm{~h}$ of incubation at $37^{\circ} \mathrm{C}$ in $5 \% \mathrm{CO}_{2}$, the non-invading cells on the upper surface were carefully removed with a cotton swab. The cells that had invaded the lower surface of the membrane were fixed with $10 \%$ formalin for $30 \mathrm{~min}$ prior to staining with crystal violet for $15 \mathrm{~min}$ at room temperature, and then counted under a light microscope at x200 magnification. The cells were counted in five random fields on each membrane. The experiments were conducted in triplicate.

Apoptosis assay. For the measurement of apoptosis, at $24 \mathrm{~h}$ post-transfection, the MDA-MB-231 and HCC-1937 cells $\left(2 \times 10^{5}\right)$ were treated with $1 \mu \mathrm{mol} / 1$ docetaxel for $36 \mathrm{~h}$. The cells were then collected in centrifuge tubes $(1,000 \mathrm{x} \mathrm{g}$, at room temperature for $5 \mathrm{~min}$ ), and washed in chilled PBS. Subsequently, the cells were re-suspended in $250 \mu \mathrm{l}$ binding buffer, and Annexin V/fluorescein isothiocyanate solution and propidium iodide (PI) solution were added to the cell suspension. Following incubation for $30 \mathrm{~min}$, the rate of apoptosis was detected by flow cytometry (FACSCanto ${ }^{\mathrm{TM}} \mathrm{II}$; BD Biosciences).

Statistical analysis. Data are presented as the mean \pm standard deviation. Two-way analysis of variance or Student's t-test was used for comparisons between groups. $\mathrm{P}<0.05$ was used to indicate a statistically significant difference. GraphPad Prism version 6.0 (GraphPad Software, Inc., La Jolla, CA, USA) or the SPSS program (IBM Corp., Armonk, NY, USA) was used to perform the statistical analyses.

\section{Results}

ASPP2 expression is downregulated in the majority of breast cancer tissues and cell lines, and is inhibited following siRNA transfection. To analyze the mRNA levels of ASPP2 expression in breast cancer tissues compared with those in para-cancerous normal tissues, the level of ASPP2 mRNA was determined by RT-qPCR. The results showed that ASPP2 mRNA expression was suppressed in a number of the breast cancer tissues compared with that in the matched normal tissues $(\mathrm{P}<0.05$; Fig. 1A). In addition, the mRNA and protein expression of ASPP2 was examined in a panel of breast cancer cell lines (BT-549, MDA-MB-231, HCC-1937, MDA-MB-468 and MCF-7) compared with the expression in the breast epithelial cell line (MCF-10A). Notably, with the exception of HCC-1937 cells, a reduction in ASPP2 mRNA expression was found in all the remaining cancer cell lines compared with that in MCF-10A, as measured by RT-qPCR ( $\mathrm{P}<0.001$; Fig. 1B). Meanwhile, western blot analysis showed that the majority of cancer cell lines (with the exception of HCC-1937 cells) 
A

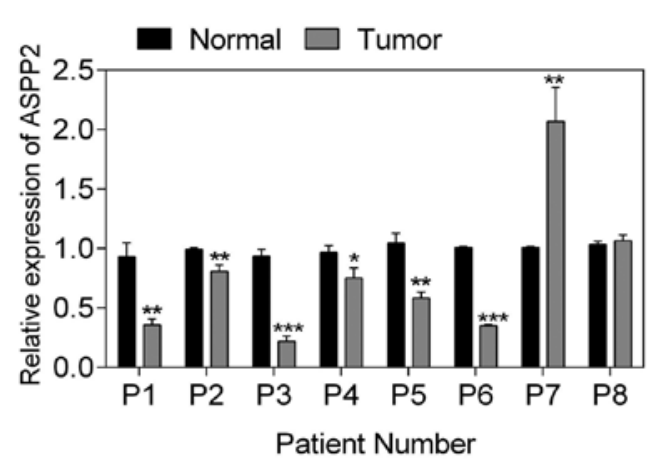

B

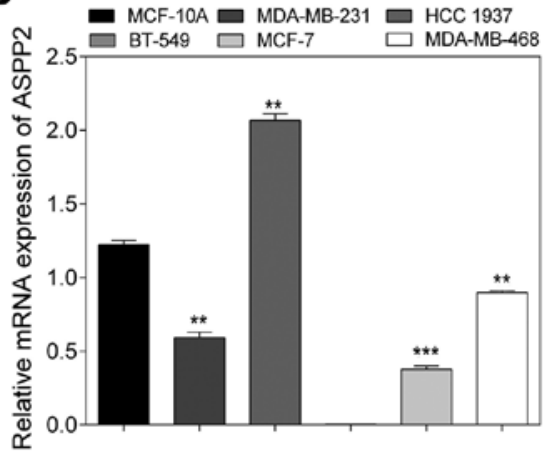

C
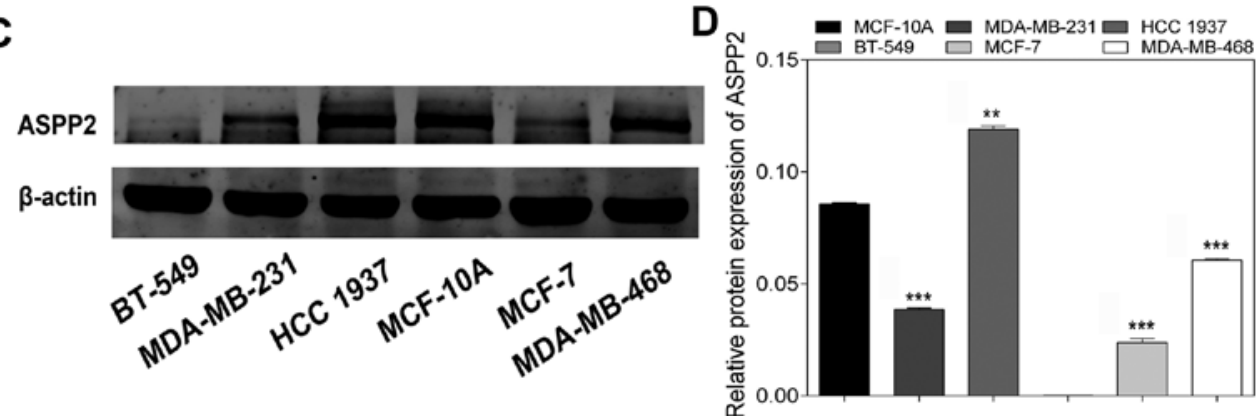

E

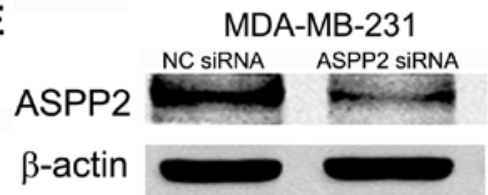

MCF-7
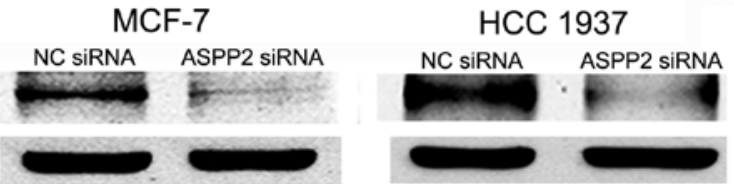

$\mathbf{F}$
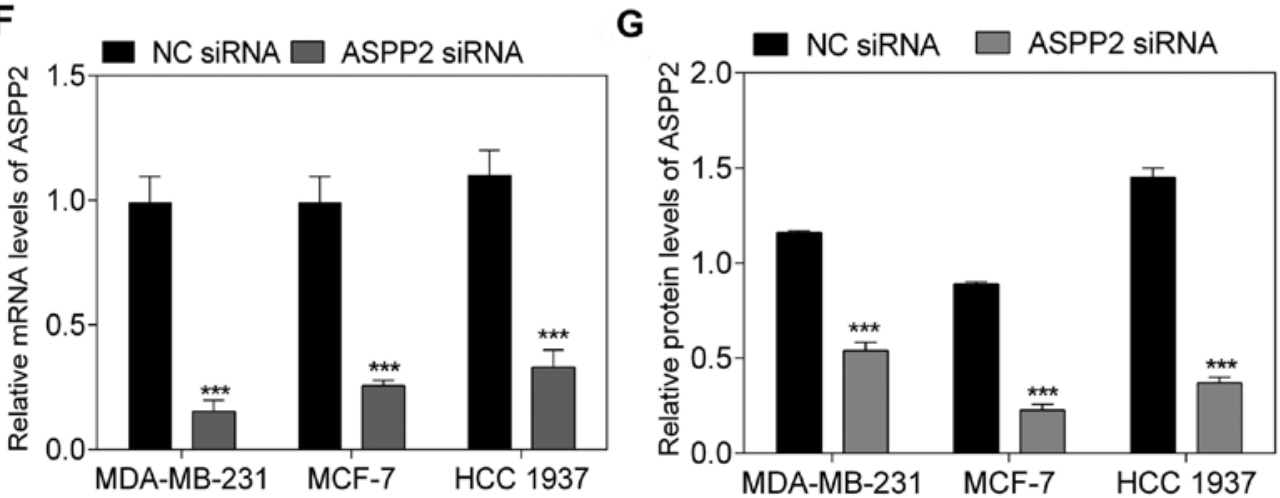

Figure 1. ASPP2 expression in breast cancer tissues and cell lines, and the efficiency of ASPP2 siRNA. (A) The relative mRNA expression of ASPP2 in breast cancer tissues. (B) The relative mRNA expression of ASPP2 in different breast cancer cell lines. (C and D) The relative protein expression of ASPP2 in five breast cancer cell lines compared with that in normal breast cells. ${ }^{*} \mathrm{P}<0.05,{ }^{* *} \mathrm{P}<0.01$ and ${ }^{* * *} \mathrm{P}<0.001$ vs. MCF-10A. (E) Silencing ASPP2 decreased the protein expression in the breast cancer cell lines. (F and G) Silencing ASPP2 decreased the relative mRNA and protein expression levels of ASPP2 in the breast cancer cell lines. Data are presented as the mean \pm standard deviation. ${ }^{*} \mathrm{P}<0.05,{ }^{* *} \mathrm{P}<0.01$ and ${ }^{* * * *} \mathrm{P}<0.001 \mathrm{vs}$. NC siRNA. ASPP2, apoptosis-stimulating p53 protein 2 ; siRNA, small interfering RNA; NC, negative control.

expressed lower levels of ASPP2 protein compared with MCF-10A, which was consistent with the tendency of the RT-qPCR results $(\mathrm{P}<0.001$; Fig. $1 \mathrm{C}$ and $\mathrm{D})$. For further investigation, ASPP2 siRNA was transfected into three different cell lines (MDA-MB-231, HCC-1937 and MCF-7 cells), and the interference effect on endogenous ASPP2 expression was validated by RT-qPCR and western blotting. Following transfection with ASPP2 siRNA, the expression of ASPP2 decreased at the mRNA and protein levels in all three different breast cancer cell lines $(\mathrm{P}<0.001$; Fig. 1E-G). Accordingly,
siRNA transfection was considered to be effective for ASPP2 silencing in breast cancer cells.

ASPP2 downregulation contributes to TNBC cell proliferation and decreases cell apoptosis. For the determination of the impact of ASPP2 on the cell viability and cell proliferation of TNBC cells, MDA-MB-231 and HCC-1937 cells were transfected with ASPP2 siRNA and NC siRNA. Subsequently, MTT and colony formation assays were performed. As shown in Fig. 2A, as determined by MTT assay, silencing ASPP2 
A MDA-MB-231
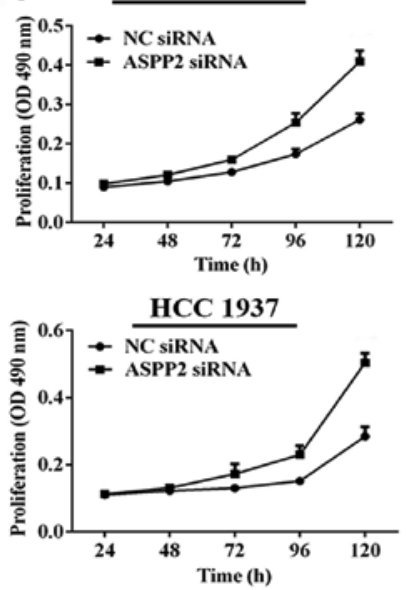

D
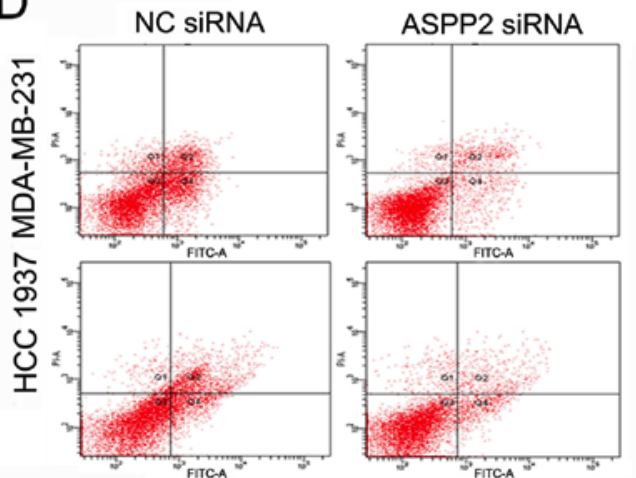

B
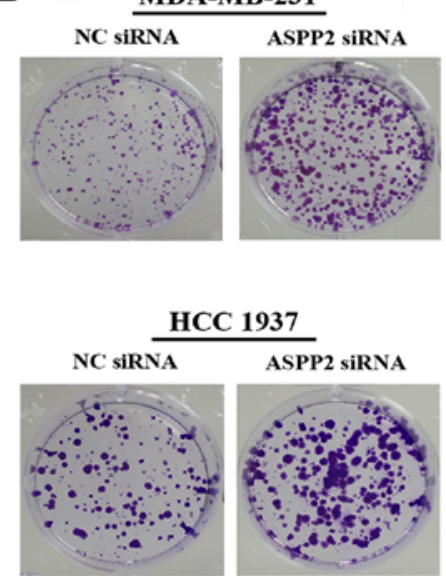

C
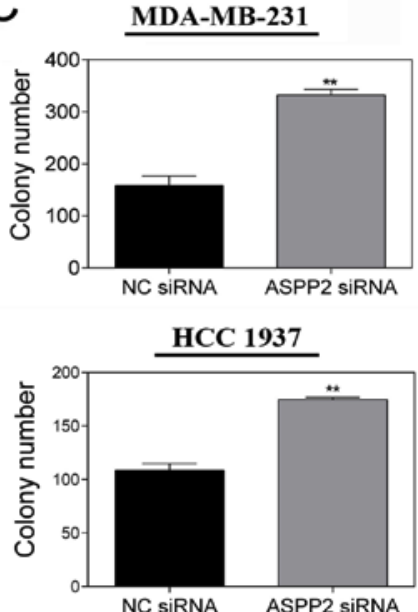

E
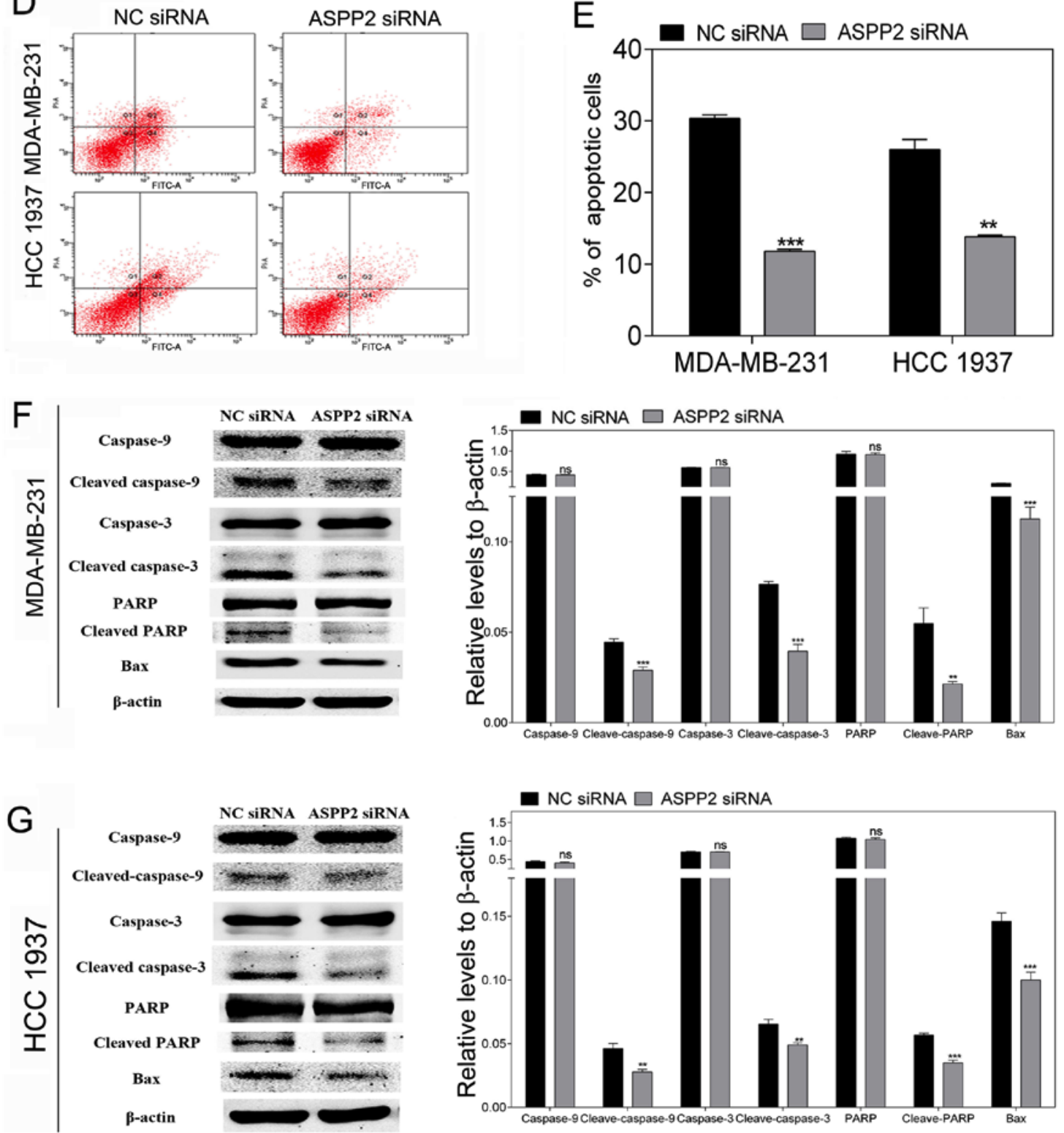

Figure 2. ASPP2 siRNA promotes proliferation and decreases apoptosis of MDA-MB-231 and HCC-1937 cells. (A) The OD values of the ASPP2 siRNA transfection groups were generally higher than those of the control groups $(\mathrm{P}<0.001)$. The OD values of the two groups increased with time $(\mathrm{P}<0.001)$. The $\mathrm{OD}$ values of the ASPP2 siRNA transfection groups increased more than that of the control groups $(\mathrm{P}<0.001)$ (two-way analysis of variance). (B and C) ASPP2 siRNA promoted the colony formation of the MDA-MB-231 and HCC-1937 cells. (D and E) ASPP2 siRNA decreased cell apoptosis. (F and G) ASPP2 siRNA regulated the expression of apoptosis-related proteins in the MDA-MB-231 and HCC-1937 cells. Data are presented as the mean \pm standard deviation. ${ }^{* *} \mathrm{P}<0.01$ and ${ }^{* * *} \mathrm{P}<0.001$ vs. NC siRNA. ASPP2, apoptosis-stimulating p53 protein 2; siRNA, small interfering RNA; NC, negative control; OD, optical density; PARP, poly (ADP-ribose) polymerase; ns, not significant. 

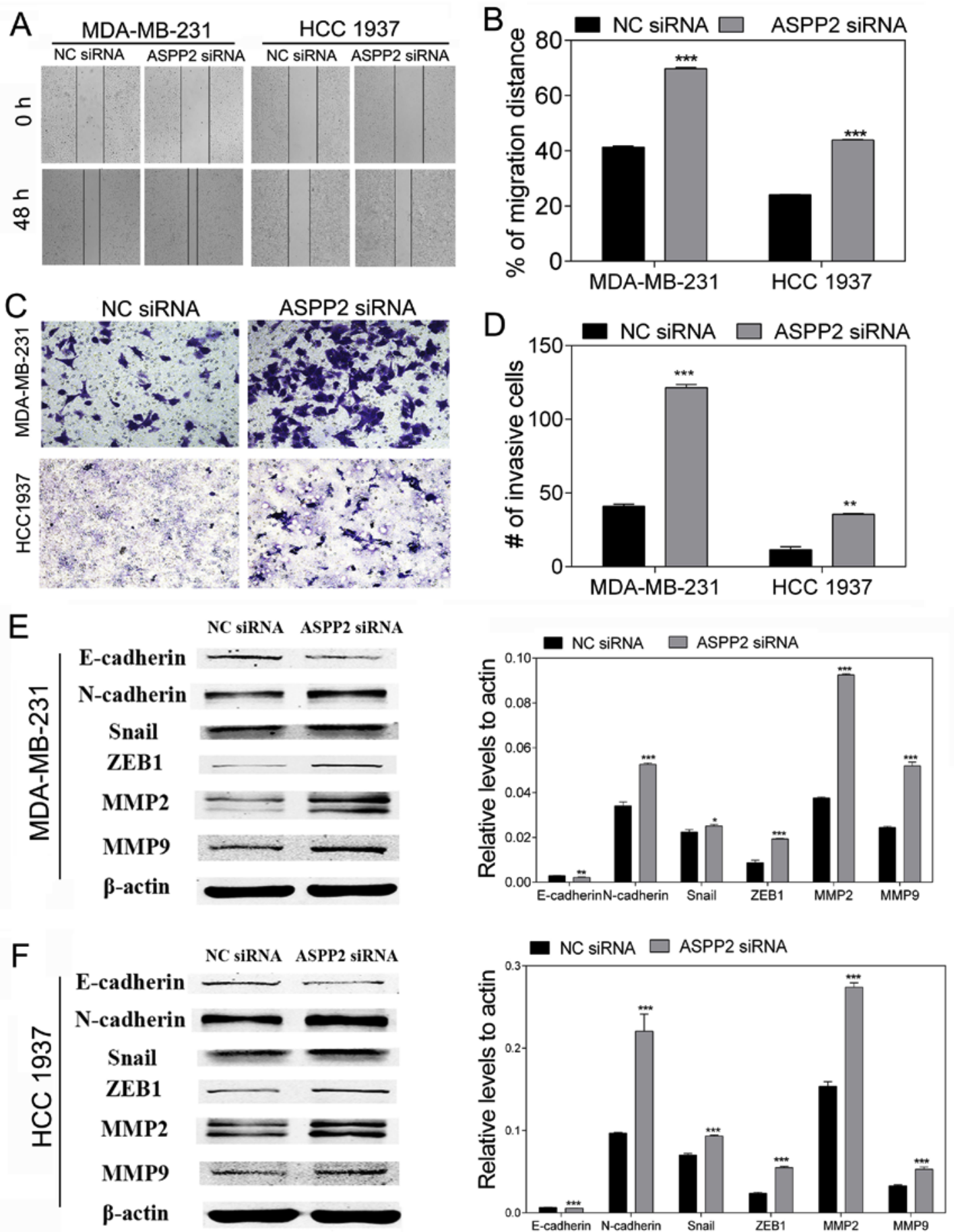

Figure 3. ASPP2 siRNA contributes to the promotion of cell migration and invasion. (A and B) ASPP2 siRNA promoted MDA-MB-231 and HCC-1937 cell migration. (C and D) ASPP2 siRNA promoted MDA-MB-231 and HCC-1937 cell invasion (crystal violet staining; x200 magnification). (E and F) ASPP2 siRNA regulated the expression of epithelial-mesenchymal transition-associated proteins in the MDA-MB-231 and HCC-1937 cells. Data are presented as the mean \pm standard deviation. ${ }^{*} \mathrm{P}<0.05,{ }^{* *} \mathrm{P}<0.01$ and ${ }^{* * *} \mathrm{P}<0.001$ vs. NC siRNA. ASPP2, apoptosis-stimulating p53 protein 2; siRNA, small interfering RNA; NC, negative control; ZEB1, zinc finger E-box-binding homeobox 1; MMP, matrix metalloproteinase.

clearly enhanced the cell proliferation in a time-dependent manner in the MDA-MB-231 and HCC-1937 cells. Likewise, the downregulation of ASPP2 caused a significant increase in the colony formation number compared with the NC siRNA transfected cells in the two cell lines $(\mathrm{P}<0.01 ;$ Fig. $2 \mathrm{~B}$ and $\mathrm{C})$. All the results indicated that ASPP2 could promote MDA-MB-231 and HCC-1937 cellular growth.

To investigate whether ASPP2 silencing could increase cell viability by reducing cell apoptosis, docetaxel $(1 \mu \mathrm{mol} / \mathrm{l})$ was added to the transfected cells to induce apoptosis following $24 \mathrm{~h}$ of transfection. Next, $36 \mathrm{~h}$ later, flow cytometric analysis was performed to analyze the apoptosis in the MDA-MB-231 and HCC-1937 cells. The total apoptosis rate of the cells was reflected by the number of early and late apoptotic cells in the Annexin $\mathrm{V}^{+} / \mathrm{PI}^{-}$and Annexin $\mathrm{V}^{+} / \mathrm{PI}^{+}$domains. As shown in Fig. 2D and $\mathrm{E}$, in comparison with the $\mathrm{NC}$ groups, the siRNA transfection group significantly decreased apoptosis. Furthermore, the western blotting results showed that 
A
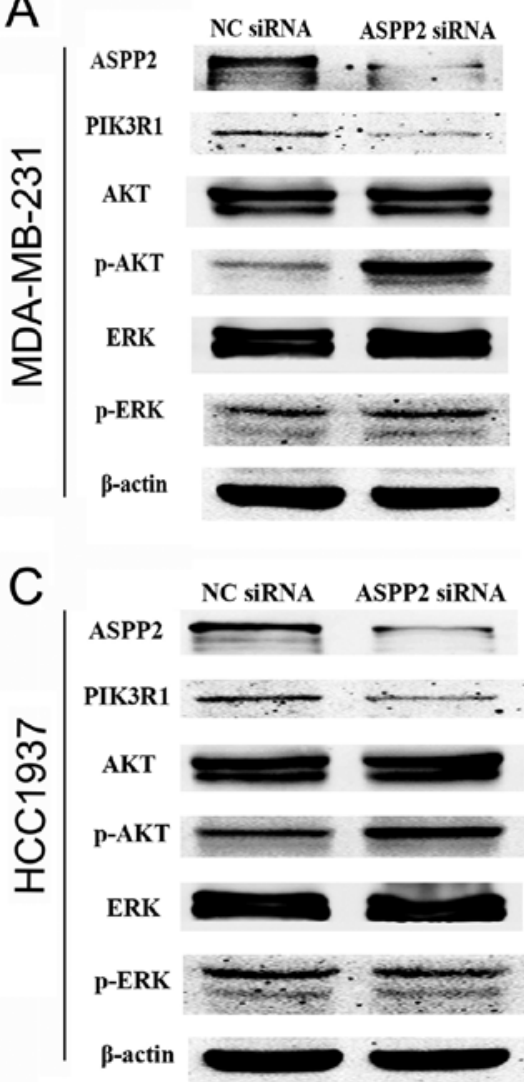

B

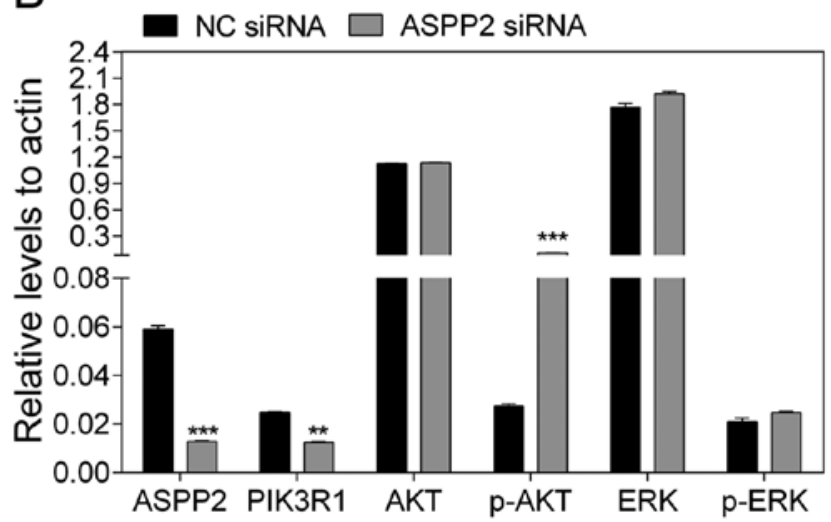

D

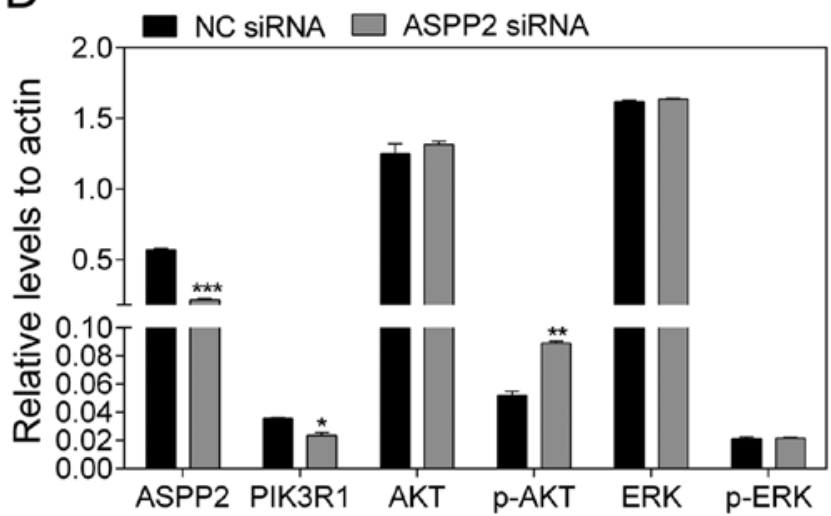

Figure 4. ASPP2 participates in regulating the PI3K/AKT pathway in breast cancer. (A and B) ASPP2 siRNA regulated PI3K/AKT-related protein levels in the MDA-MB-231 cells. (C and D) ASPP2 siRNA regulated PI3K/AKT-related protein levels in the HCC-1937 cells. Data are presented as the mean \pm standard deviation. ${ }^{*} \mathrm{P}<0.05,{ }^{* *} \mathrm{P}<0.01$ and ${ }^{* * *} \mathrm{P}<0.001$ vs. NC siRNA. ASPP2, apoptosis-stimulating p53 protein 2; siRNA, small interfering RNA; NC, negative control; p-, phosphorylated; AKT, protein kinase B; PIK3R1, phosphoinositide-3-kinase regulatory subunit 1; ERK, extracellular signal-regulated kinases.

the levels of apoptosis-related proteins, including cleaved caspase-9, cleaved caspase-3, cleaved PARP and Bax, were significantly decreased compared with those of the NC groups (Fig. 2F and G). Caspase-9 is at the top of the caspase cascade activation response, as the most important promoter and key protease of mitochondrial apoptotic pathway, the activation of which can further activate the downstream Caspase family and then promote cell apoptosis (21). Bax can enhance the permeability of the mitochondrial membrane for entry of cytochrome $c$ into the cytoplasm and then promote cell apoptosis (22). All the data indicated that ASPP2 silencing promoted cell proliferation and reduced cell apoptosis, perhaps through the mitochondrial death pathway.

ASPP2 downregulation accelerates cell migration, invasion and EMT in TNBC cells. To investigate the role of ASPP2 in cell migration and invasion, wounding-healing and Transwell invasion assays were performed. The wound-healing assay showed that ASPP2 silencing significantly promoted the migration ability of the MDA-MB-231 and HCC-1937 cells compared with the NC (both $\mathrm{P}<0.001$; Fig. $3 \mathrm{~A}$ and B). The Transwell invasion assay showed that ASPP2 silencing increased the invasion ability of the MDA-MB-231 and HCC-1937 cells ( $\mathrm{P}<0.01$ and $\mathrm{P}<0.001$; Fig. $3 \mathrm{C}$ and $\mathrm{D})$. These results indicated a direct association between ASPP2 and the motility of TNBC cells. On the basis of the cell functional study, research on the EMT-related proteins was performed using western blot to further investigate the effect of ASPP2 on the migration and invasion mechanism. Following depletion of ASPP2, the expression of representative epithelial marker E-cadherin significantly decreased, whereas the expression of mesenchymal marker $\mathrm{N}$-cadherin and other key markers, including Snail and ZEB1, all increased. Furthermore, the levels of MMP2 and MMP9, which are involved in EMT, also increased (Fig. 3E and F). Taken together, these results suggest that ASPP2 silencing may be responsible for EMT development, and this serves a vital role in the progression of breast cancer

ASPP2 influences the PI3K/AKT signaling pathway. Activation of the PI3K/AKT signaling pathway is regarded as a crucial emblem in breast cancer that is associated with its development, progress and metastatic spread (23). To further validate whether ASPP2 is involved in the p53-independent pathway in TNBC, the effect of ASPP2 on the PI3K/AKT pathway was investigated. The important molecular markers associated with the PI3K/AKT pathway were then detected. As shown in Fig. 4, the downregulation of ASPP2 resulted in the decreased expression of PIK3R1 (p85 $\alpha$ ) and the increased expression of p-AKT, whereas it had no influence on the expression of p-ERK.

PIK3R1 (p85 $\alpha$ ) is the regulatory subunit of PI3K and negatively regulates the PI3K pathway (24). The present results suggested that the downregulation of ASPP2 was able to activate the PI3K/AKT pathway in TNBC cells. 


\section{Discussion}

TNBC is considered to be a cancer with one of the worst prognoses of all the breast cancer subtypes. A growing body of evidence has shown that the aberrant expression of certain genes may result in tumor progression and metastasis. Previous studies showed that ASPP2 was suppressed in breast cancer tissues, and that the low expression of ASPP2 predicted a poor prognosis in pancreatic cancer $(15,25)$. Consistent with these results, the present study found decreased ASPP2 mRNA levels in breast cancer tissues compared with those in normal para-cancerous tissues. In addition, ASPP2 expression was diminished in the majority of the breast cancer cell lines, with the exception of the HCC-1937 cell line, at the mRNA and protein levels. This finding suggests that loss of ASPP2 may profoundly affect the pathogenesis of breast cancer. The abnormally high expression in HCC-1937 cells attracted was notable with regard to whether it had an unexpected role in such TNBC cells.

Through knockdown of ASPP2 by specific siRNAs, cell function findings demonstrated that the downregulation of ASPP2 promoted cell proliferation and increased the migration and invasion abilities in HCC-1937 cells, in accordance with the results in MDA-MB-231 cells; this affirmed the inhibitory role of ASPP2 in TNBC and refuted the possibility of ASPP2 acting as an oncogene and causing the high expression. The increased expression of ASPP2 may arise from the failure to compensate for the abnormal expression of certain genes. The BRCA1 DNA repair-associated gene primarily promotes DNA repair in response to DNA damage, and HCC-1937 cells are deficient in it (26). By binding to p53, ASPP2 enables p53 to selectively upregulate the expression of pro-apoptotic genes in response to DNA damage (27), and its expression can be upregulated in response to DNA damage (28). Whether the expression of the two genes are linked remains to be further investigated. In contrast to previous results, one previous study showed that ASPP2 expression was downregulated in wild-type p53 tumor cells due to promoter hypermethylation (29). It was found that ASPP2 expression depends on the methylation status, which remains to be assessed in breast cancer cell lines for a better understanding of the mechanism of ASPP2 expression.

ASPP2, as an anti-oncogene, functions primarily in stimulating apoptosis and enhancing the expression of proapoptotic genes. In previous studies, ASPP2 downregulation was found to be a vital component of microRNA-548-3p, inducing cell proliferation and reducing cell apoptosis (30). In addition, ASPP2 suppressed cell autophagy and facilitated oxaliplatin-induced colorectal cancer cell apoptosis (31). In the same way, reduced apoptosis by silencing ASPP2 in the TNBC cells was observed in the present study. Notably, it was also found that the decreased apoptosis was accompanied by the deactivation of the caspase family and Bax in the TNBC cells. The apoptosis pathway is traditionally divided into two types: The death-receptor (extrinsic) pathway represented by caspase- 8 and cellular FADD-like IL-1 $\beta$-converting enzyme-inhibitory protein, and the mitochondrial (intrinsic) pathway represented by caspase- 9 and Bax (32). The present study confirmed that ASPP2 induced apoptosis via the mitochondrial pathway, supporting earlier findings (33). Nevertheless, whether the death-receptor pathway or other mechanisms influenced ASPP2-induced apoptosis remains to be evaluated.
EMT serves as a key promoter of the aggression, invasion and metastasis of cancer, characterized by the loss or reduction of epithelial markers (E-cadherin and cytokeratins), together with the overexpression of mesenchymal markers (N-cadherin and Vimentin) (34). ASPP2 can suppress EMT by preventing $\beta$-catenin from entering the nucleus to accelerate ZEB1 expression in accordance with its limiting ability on oncogenic RAS, and the low level of ASPP2 is indicative of poor patient survival and positive lymph node status in numerous cancer types (12). Protein phosphatase $\mathrm{Mg}^{2+} / \mathrm{Mn}^{2+}$-dependent 1D was found to promote cell migration and invasion in pancreatic cancer via the Wnt/ $\beta$-catenin pathway on the basis of ASPP2 reduction (35). The present results showed that the depletion of ASPP2 decreased the expression of E-cadherin in TNBC cells, whereas the expression of other markers, including N-cadherin, ZEB1, Snail, MMP2 and MMP9, was increased, all of which predicted the EMT process occurring followed by a gain in the ability of migration and invasion. These findings provide the possible mechanism by which ASPP2 affects growth and metastasis in TNBC.

The PI3K/AKT pathway is one of the most frequently $(\sim 50 \%)$ dysregulated pathways in TNBC that is caused by key gene mutants, leading to the overactivation of AKT or the functional loss of regulation factors, including phosphatase and tensin homolog protein (36). PI3K inhibitors were reported to provide novel insights into TNBC therapy, which has been confirmed to have more notable efficacy on the tumor volume and mitotic activity in TNBC xenografts compared with that in the luminal-like xenografts (37). To the best of our knowledge, ASPP2 serves key roles not only in a p53-dependent manner to regulate apoptosis, but also with involvement in the other p53-independent pathways, including the nuclear factor-кB (38), Hippo $(11,39,40)$ and Wnt/ $\beta$-catenin (35) pathways. As ASPP2 has almost no influence on mutant-p53, whereas p53 mutation is a common occurrence in TNBC, the p53-independent role of ASPP2 deserves more attention. In consideration of the importance of the PI3K/AKT pathway in TNBC, we hypothesized that ASPP2 was associated with the PI3K/AKT pathway, acting in a p53-independent manner in TNBC. The downregulation of ASPP2 was demonstrated to result in the abatement of PIK3R1 (p85 $\alpha$ ). p85 $\alpha$ has been reported to have an inhibitory effect on the PI3K pathway, the downregulation of which increased the p-AKT levels accordingly, promoting breast cancer cell growth, migration and invasion (41). Consistent with this previous study, the p-AKT level in the present study was affirmed to increase when ASPP2 was silenced, causing a reduction in $\mathrm{p} 85 \alpha$, whereas the p-ERK level exhibited no significant change. In one sense, this finding confirmed the aforementioned hypothesis. However, another previous study showed that ASPP2 potentiated p-ERK activation other than p-AKT activation through stimulating Ras signaling in primary human fibroblasts (9). The two opposite results can possibly be explained by the fact that the PI3K/AKT pathway is more involved than Ras signaling in TNBC, resulting in the selective impact of ASPP2 on the PI3K/AKT pathway. However, the detailed mechanism between ASPP 2 and the PI3K/AKT pathway in TNBC requires further investigation.

Taken together, the present study results have provided evidence that ASPP2 has an inhibitory influence on TNBC growth and metastasis, and that this may rely on using the PI3K/AKT pathway in a p53-independent manner. These find- 
ings may assist in the development of more valuable strategies for the treatment of TNBC.

\section{Acknowledgements}

We sincerely thank all the teachers at the Central Laboratory of the Shanghai Tenth People's Hospital for their help and support.

\section{Funding}

This study was supported by grant no. 201640097 from the Shanghai Municipal Health Bureau of Shanghai, China, and grant no. 82172240 from the National Natural Science Foundation of China.

\section{Availability of data and materials}

The datasets used during the present study are available from the corresponding author upon reasonable request.

\section{Authors' contributions}

TW, LF and HS conceived and designed the study. TW, DX and $\mathrm{BZ}$ performed the experiments. TW, KH and JH wrote the paper. HX, CW, YD and CJ reviewed and edited the manuscript. YD, JH and CJ made contributions to the aquisition of patients' data. $\mathrm{HX}, \mathrm{KH}$ and $\mathrm{CW}$ analyzed and interpreted the data. All authors read and approved the manuscript and agree to be accountable for all aspects of the research in ensuring that the accuracy or integrity of any part of the work are appropriately investigated and resolved.

\section{Ethics approval and consent to participate}

All experimental protocols were approved by the Institutional Ethics Committees of Shanghai Tenth People's Hospital Affiliated to Tongji University (Shanghai, China).

\section{Consent for publication}

Not applicable.

\section{Competing interests}

The authors state that they have no competing interests.

\section{References}

1. Miller KD, Siegel RL, Lin CC, Mariotto AB, Kramer JL, Rowland JH, Stein KD, Alteri R and Jemal A: Cancer treatment and survivorship statistics, 2016. CA Cancer J Clin 66: 271-289, 2016.

2. Pal SK, Childs BH and Pegram M: Triple negative breast cancer: Unmet medical needs. Breast Cancer Res Treat 125: 627-636, 2011

3. Mayer IA, Abramson VG, Lehmann BD and Pietenpol JA: New strategies for triple-negative breast cancer--deciphering the heterogeneity. Clin Cancer Res 20: 782-790, 2014.

4. Perou CM: Molecular stratification of triple-negative breast cancers. Oncologist 16 (Suppl 1): 61-70, 2011.

5. Takahashi N, Kobayashi S, Jiang X, Kitagori K, Imai K, Hibi Y and Okamoto T: Expression of 53BP2 and ASPP2 proteins from TP53BP2 gene by alternative splicing. Biochem Biophys Res Commun 315: 434-438, 2004.
6. Samuels-Lev Y, O'Connor DJ, Bergamaschi D, Trigiante G, Hsieh JK, Zhong S, Campargue I, Naumovski L, Crook T and $\mathrm{Lu}$ X: ASPP proteins specifically stimulate the apoptotic function of p53. Mol Cell 8: 781-794, 2001.

7. Ahn J, Byeon IJ, Byeon CH and Gronenborn AM: Insight into the structural basis of pro- and antiapoptotic p53 modulation by ASPP proteins. J Biol Chem 284: 13812-13822, 2009.

8. Trigiante G and Lu X: ASPP [corrected] and cancer. Nat Rev Cancer 6: 217-226, 2006.

9. Wang Z, Liu Y, Takahashi M, Van Hook K, KampaSchittenhelm KM, Sheppard BC, Sears RC, Stork PJ and Lopez CD: $\mathrm{N}$ terminus of ASPP2 binds to Ras and enhances Ras/Raf/MEK/ERK activation to promote oncogene-induced senescence. Proc Natl Acad Sci USA 110: 312-317, 2013.

10. Liu Z, Qiao L, Zhang Y, Zang Y, Shi Y, Liu K, Zhang X, $\mathrm{Lu}$ X, Yuan L, Su B, et al: ASPP2 plays a dual role in gp120induced autophagy and apoptosis of neuroblastoma cells. Front Neurosci 11: 150, 2017.

11. Royer C, Koch S, Qin X, Zak J, Buti L, Dudziec E, Zhong S, Ratnayaka I, Srinivas S and Lu X: ASPP2 links the apical lateral polarity complex to the regulation of YAP activity in epithelial cells. PLoS One 9: e111384, 2014.

12. Wang Y, Bu F, Royer C, Serres S, Larkin JR, Soto MS, Sibson NR, Salter V, Fritzsche F, Turnquist C, et al: ASPP2 controls epithelial plasticity and inhibits metastasis through $\beta$-catenin-dependent regulation of ZEB1. Nat Cell Biol 16: 1092-1104, 2014.

13. Zhao J, Wu G, Bu F, Lu B, Liang A, Cao L, Tong X, Lu X, Wu M and Guo Y: Epigenetic silence of ankyrin-repeat-containing, SH3-domain-containing, and proline-rich-region- containing protein 1 (ASPP1) and ASPP2 genes promotes tumor growth in hepatitis B virus-positive hepatocellular carcinoma. Hepatology 51: 142-153, 2010.

14. Wang X, Yu M, Zhao K, He M, Ge W, Sun Y, Wang Y, Sun H and $\mathrm{Hu}$ Y: Upregulation of MiR-205 under hypoxia promotes epithelial-mesenchymal transition by targeting ASPP2. Cell Death Dis 7: e2517, 2016.

15. Song B, Bian Q, Zhang YJ, Shao CH, Li G, Liu AA, Jing W, Liu R, Zhou YQ, Jin G, et al: Downregulation of ASPP2 in pancreatic cancer cells contributes to increased resistance to gemcitabine through autophagy activation. Mol Cancer 14: 177, 2015.

16. Jabbour-Leung NA, Chen X, Bui T, Jiang Y, Yang D, Vijayaraghavan S, McArthur MJ, Hunt KK and Keyomarsi K: Sequential combination therapy of CDK inhibition and doxorubicin is synthetically lethal in p53-mutant triple-negative breast cancer. Mol Cancer Ther 15: 593-607, 2016.

17. Slee EA and Lu X: The ASPP family: deciding between life and death after DNA damage. Toxicol Lett 139: 81-87, 2003.

18. Joerger AC, Ang HC, Veprintsev DB, Blair CM and Fersht AR: Structures of $\mathrm{p} 53$ cancer mutants and mechanism of rescue by second-site suppressor mutations. J Biol Chem 280: 16030-16037, 2005.

19. Barnes L, Eveson JW, Reichart P and Sidransky D: WHO Classification of Tumours, 2005.

20. Livak KJ and Schmittgen TD: Analysis of relative gene expression data using real-time quantitative PCR and the 2(- $\Delta \Delta C(T))$ method. Methods 25: 402-408, 2001.

21. Bratton SB and Salvesen GS: Regulation of the Apaf-1-caspase-9 apoptosome. J Cell Sci 123: 3209-3214, 2010.

22. Gross A, McDonnell JM and Korsmeyer SJ: BCL-2 family members and the mitochondria in apoptosis. Genes Dev 13: 1899-1911, 1999.

23. Azim HA, Kassem L, Treilleux I, Wang Q, El Enein MA, Anis SE and Bachelot T: Analysis of PI3K/mTOR pathway biomarkers and their prognostic value in women with hormone receptor-positive, HER2-Negative Early Breast Cancer. Transl Oncol 9: 114-123, 2016.

24. Luo J and Cantley LC: The negative regulation of phosphoinositide 3-kinase signaling by $\mathrm{p} 85$ and it's implication in cancer. Cell Cycle 4: 1309-1312, 2005 .

25. Van Hook K, Wang Z, Chen D, Nold C, Zhu Z, Anur P, Lee HJ, Yu Z, Sheppard B, Dai MS, et al: $\triangle \mathrm{N}$-ASPP2, a novel isoform of the ASPP2 tumor suppressor, promotes cellular survival. Biochem Biophys Res Commun 482: 1271-1277, 2017.

26. Yoshida K and Miki Y: Role of BRCA1 and BRCA2 as regulators of DNA repair, transcription, and cell cycle in response to DNA damage. Cancer Sci 95: 866-871, 2004.

27. Slee EA and Lu X: The ASPP family: Deciding between life and death after DNA damage. Toxicol Lett 139: 81-87, 2003.

28. Bergamaschi D, Samuels Y, Jin B, Duraisingham S, Crook T and $\mathrm{Lu} \mathrm{X}$ : ASPP1 and ASPP2: Common activators of p53 family members. Mol Cell Biol 24: 1341-1350, 2004. 
29. Liu ZJ, Lu X, Zhang Y, Zhong S, Gu SZ, Zhang XB, Yang X and Xin HM: Downregulated mRNA expression of ASPP and the hypermethylation of the 5'-untranslated region in cancer cell lines retaining wild-type p53. FEBS Lett 579: 1587-1590, 2005.

30. Song Q, Song J, Wang Q, Ma Y, Sun N, Ma J, Chen Q, Xia G, Huo Y, Yang L, et al: miR-548d-3p/TP53BP2 axis regulates the proliferation and apoptosis of breast cancer cells. Cancer Med 5: 315-324,2016.

31. Shi Y, Han Y, Xie F, Wang A, Feng X, Li N, Guo H and Chen D: ASPP2 enhances oxaliplatin (L-OHP)-induced colorectal cancer cell apoptosis in a p53-independent manner by inhibiting cell autophagy. J Cell Mol Med 19: 535-543, 2015.

32. Hengartner MO: The biochemistry of apoptosis. Nature 407: 770-776, 2000

33. Liu K, Jiang T, Ouyang Y, Shi Y, Zang Y, Li N, Lu S and Chen D: Nuclear EGFR impairs ASPP2-p53 complex-induced apoptosis by inducing SOS1 expression in hepatocellular carcinoma. Oncotarget 6: 16507-16516, 2015.

34. Sarrió D, Rodriguez-Pinilla SM, Hardisson D, Cano A, Moreno-Bueno G and Palacios J: Epithelial-mesenchymal transition in breast cancer relates to the basal-like phenotype. Cancer Res 68: 989-997, 2008.

35. Wu B, Guo BM, Kang J, Deng XZ, Fan YB, Zhang XP and Ai KX: PPM1D exerts its oncogenic properties in human pancreatic cancer through multiple mechanisms. Apoptosis 21: 365-378, 2016.
36. Yuan TL and Cantley LC: PI3K pathway alterations in cancer: Variations on a theme. Oncogene 27: 5497-5510, 2008.

37. Moestue SA, Dam CG, Gorad SS, Kristian A, Bofin A Mælandsmo GM, Engebråten O, Gribbestad IS and Bjørkøy G: Metabolic biomarkers for response to PI3K inhibition in basallike breast cancer. Breast Cancer Res 15: R16, 2013.

38. Song X, Du J, Zhu W, Jin P and Ma F: Identification and characterization of an apoptosis-stimulating protein of p53 (ASPP) gene from Branchiostoma belcheri: Insights into evolution of ASPP gene family. Fish Shellfish Immunol 49: 268-274, 2016.

39. Gao K, An J, Zhang Y, Jin X, Ma J, Peng J, Tang Y, Yu L, Zhang P and Wang C: The E3 ubiquitin ligase Itch and Yap1 have antagonistic roles in the regulation of ASPP2 protein stability. FEBS Lett 589: 94-101, 2015.

40. Liu CY, Lv X, Li T, Xu Y, Zhou X, Zhao S, Xiong Y, Lei QY and Guan KL: PP1 cooperates with ASPP2 to dephosphorylate and activate TAZ. J Biol Chem 286: 5558-5566, 2011

41. Yan LX, Liu YH, Xiang JW, Wu QN, Xu LB, Luo XL, Zhu XL, Liu C, Xu FP, Luo DL, et al: PIK3R1 targeting by miR-21 suppresses tumor cell migration and invasion by reducing PI3K/AKT signaling and reversing EMT, and predicts clinical outcome of breast cancer. Int J Oncol 48: 471-484, 2016. 